\title{
Nuclear factor- $\kappa B$ decoy attenuates neuronal damage after global brain ischemia: A future strategy for brain protection during circulatory arrest
}

Takayoshi Ueno, MDa

Yoshiki Sawa, MDa

Satoru Kitagawa-Sakakida, MDa

Motonobu Nishimura, MDa

Ryuichi Morishita, MD ${ }^{\mathrm{b}}$

Yasufumi Kaneda, $\mathrm{MD}^{\mathrm{b}}$

Eiji Kohmura, MDC

Toshiki Yoshimine, MDc

Hikaru Matsuda, MD
From the Division of Cardiovascular Surgery, Department of Surgery, ${ }^{\text {a }}$ Department of Gene Therapy, ${ }^{b}$ and Department of Neurosurgery, ${ }^{\mathrm{c}}$ Osaka University Graduate School of Medicine, Suita, Osaka, Japan.

Received for publication Nov 22, 2000; revisions requested Jan 25, 2001; revisions received March 1, 2001; accepted for publication March 19, 2001.

Address for reprints: Yoshiki Sawa, MD, Division of Cardiovascular Surgery, Department of Surgery, Osaka University Graduate School of Medicine, 2-2 Yamadaoka, Suita, Osaka 565-0871, Japan (E-mail: sawa@surg1.med.osaka-u.ac.jp).

J Thorac Cardiovasc Surg 2001;122:720-7

Copyright (C) 2001 by The American Association for Thoracic Surgery

$0022-5223 / 2001 \$ 35.00+0 \quad \mathbf{1 2 / 1 / 1 1 5 9 1 7}$

doi: $10.1067 / \mathrm{mtc} .2001 .115917$
Objectives: Recent studies have reported that cis element decoy oligodeoxynucleotides against nuclear factor- $\mathrm{\kappa B}$ block the activation of genes that mediate ischemic injury. To improve brain protection during circulatory arrest in cardiac surgery, we evaluated the efficacy of nuclear factor- $\mathrm{\kappa B}$ decoy oligodeoxynucleotides in preventing neuronal damage after global brain ischemia.

Methods: Hemagglutinating virus of Japan-liposome complex with fluorescein isothiocyanate-labeled nuclear factor- $\mathrm{\kappa B}$ decoy oligodeoxynucleotides was injected through the carotid artery during 20 minutes of global brain ischemia in rats to evaluate the efficacy of transfecting the decoy oligodeoxynucleotides. The messenger RNA levels of several factors related to ischemia-reperfusion injury in the hippocampus were estimated by a real-time polymerase chain reaction method 1 hour after reperfusion. Neuronal damage was evaluated by terminal deoxynucleotidyl transferase-mediated deoxyuridine triphosphate nick end labeling staining and by using immunohistochemical study of microtubule-associated protein 2 in the hippocampus CA-1 region 7 days after ischemia.

Results: Introduction of the nuclear factor- $\mathrm{\kappa B}$ decoy oligodeoxynucleotides into rat brain neurons through the carotid artery during global brain ischemia was markedly successful. The polymerase chain reaction study showed that the transfected nuclear factor- $\mathrm{\kappa B}$ decoy oligodeoxynucleotides effectively inhibited the expression of tumor necrosis factor $\alpha$ interleukin $1 \beta$ and intracellular adhesion molecule 1 messenger RNA 1 hour after global brain ischemia. Terminal deoxynucleotidyl transferase-mediated deoxyuridine triphosphate nick end labeling staining and microtubule-associated protein 2 immunohistochemistry showed that the transfected nuclear factor- $\kappa \mathrm{B}$ decoy oligodeoxynucleotides significantly attenuated the neuronal damage 7 days after global brain ischemia.

Conclusions: Therapeutic transfection of nuclear factor- $\kappa \mathrm{B}$ decoy oligodeoxynucleotides during brain ischemia may be useful for attenuating neuronal damage, suggesting a strategy for cerebral protection against global ischemia.

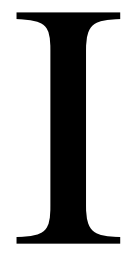

$\mathrm{n}$ the field of cardiac surgery, circulatory arrest is commonly used as the support technique in patients with aortic aneurysmal changes or in neonates with complex congenital anomalies. However, several complications related to circulatory arrest are still unresolved, and longer duration of circulatory arrest results in a higher incidence of neurologic sequelae. ${ }^{1}$ During circulatory arrest, the whole body, including the brain, is ischemic, and prolonged ischemia leads 
to necrosis of the neurons. Moreover, brain neurons, especially those in the hippocampus, will die 5 to 7 days after even a few minutes of ischemia, a phenomenon called delayed neuronal death. ${ }^{2}$ Even when techniques such as deep hypothermia are used to protect the brain against ischemic injury, 45 to 60 minutes is the outer limit for maintaining circulatory arrest, and deep hypothermia is associated with several risks, including increased bleeding, blood transfusion, and a decline of immunity. ${ }^{3}$ The development of better techniques for brain protection against both neuronal necrosis and delayed neuronal death resulting from ischemic insult is desired to improve the morbidity of operations for aortic and congenital heart disease.

Recent studies have implicated the activation of nuclear factor- $\kappa \mathrm{B}(\mathrm{NF}-\kappa \mathrm{B})$, a crucial transcriptional factor, in neuronal damage after cerebral ischemia. ${ }^{4-6} \mathrm{NF}-\kappa \mathrm{B}$ is a transcriptional activator of many genes whose expression is related to ischemia-reperfusion injury, such as cytokines (tumor necrosis factor $\alpha[$ TNF- $\alpha]$ and interleukin $1 \beta[\mathrm{IL}-1 \beta])^{7}$ and adhesion molecules (intracellular adhesion molecule 1 [ICAM-1]). ${ }^{8}$ Furthermore, inhibitors of NF- $\kappa \mathrm{B}$ activation, such as aspirin, seem to block ischemic injury in neurons. ${ }^{9}$ It has been reported that the transfection of decoy oligodeoxynucleotides (ODNs) blocks the transcriptional activation of cytokines and adhesion molecules, ${ }^{10}$ and we previously reported the efficacy of transfecting NF- $\kappa \mathrm{B}$ decoy ODNs to prevent ischemic reperfusion injury in the heart. ${ }^{11,12}$

On the basis of these findings, we hypothesized that transfecting NF- $\kappa \mathrm{B}$ decoy ODNs into neurons would prevent neuronal damage after global brain ischemia and that $\mathrm{NF}-\kappa \mathrm{B}$ decoy ODNs could provide a new cerebroprotective therapy. The present study was designed to examine whether transfecting NF- $\mathrm{KB}$ decoy ODNs into the brain through the carotid artery could attenuate the damage to neurons after global brain ischemia in a rat model. Our goal is to develop a new cerebroprotective agent for use during global brain ischemia including circulatory arrest in cardiovascular surgery.

\section{Methods \\ Preparation of Hemagglutinating Virus of Japan-Liposome Complexes}

Hemagglutinating virus of Japan (HVJ)-liposome complexes were prepared as described previously. ${ }^{11}$ In brief, phosphatidylserine, phosphatidylcholine, and cholesterol were mixed in a weight ratio of 1:4.8:2. The lipid mixture $(10 \mathrm{mg})$ was deposited on the sides of a flask by removing the solvent, tetrahydrofuran, in a rotary evaporator. The dried lipid was hydrated in $200 \mu \mathrm{L}$ of a balanced salt solution with $200 \mu \mathrm{g}$ of ODNs. Liposomes were prepared by shaking and sonication. The liposome suspension $(0.5 \mathrm{~mL}$ containing $10 \mathrm{mg}$ lipid) was mixed with inactivated HVJ (10,000 hemagglutinating units) in a total volume of $4 \mathrm{~mL}$ of balanced salt solution. The mixture was incubated at $4^{\circ} \mathrm{C}$ for 5 minutes and then for 30 minutes at $37^{\circ} \mathrm{C}$ with gentle shaking. Free $\mathrm{HVJ}$ was removed from the HVJ-liposomes by means of sucrose density gradient centrifugation. The top layer of the sucrose gradient was collected for use. The sequences of the phosphorothioate ODNs were as follows: NF- $\kappa$ B decoy ODNs, 5'-CCTTGAAGGGATTTCCCTCC-3' and 3'-GGAACTTCCCTAAAGGGAGG-5'; scrambled decoy ODNs, 5'-TTGCCGTACCTGACTTAGCC-3' and 3'-AACGGCATCCACTGAATCGG-5'.

\section{Global Brain Ischemia Model}

To establish the rat global brain ischemia-reperfusion model, we modified the subclavian-carotid occlusion technique. ${ }^{13}$ Male Sprague-Dawley rats weighing 300 to $350 \mathrm{~g}$ were used. All animals received humane care in compliance with the "Guide for the Care and Use of Laboratory Animals" prepared by the Institute of Laboratory Animal Resources in the Osaka University Medical School. Each rat was anesthetized with $50 \mathrm{mg} / \mathrm{kg}$ pentobarbital administered intraperitoneally and intubated into the mouth. The rodent ventilator was set at $10 \mathrm{~mL} / \mathrm{kg}$ volume and 50 to 60 strokes/min to maintain a $\mathrm{PCO}_{2}$ of $35 \mathrm{~mm} \mathrm{Hg}$. During the experiment, rats were warmed on heating blankets at $36^{\circ} \mathrm{C}$, except for the brain. After thoracotomy, the left lobe of the thymus was removed. The aortic arch was identified, and the innominate artery, left common carotid artery, and left subclavian artery were snared with 50 nylon sutures. The right common carotid artery was exposed in the neck and cannulated with a polyethylene tube (PE10, Becton Dickinson Company, Franklin Lakes, NJ). Global brain ischemia was induced by clamping all 3 sutured arteries for 20 minutes. Immediately after the arteries were clamped, the HVJ-liposome complex containing either the NF- $\kappa \mathrm{B}$ decoy ODNs (NF decoy group) or the scrambled decoy ODNs (S decoy group) was infused into the right carotid artery to perfuse the brain tissue. These drugs were stored at $4^{\circ} \mathrm{C}$, and $2 \mathrm{~mL}$ per animal was infused. The pharyngeal temperature fell from $35.2^{\circ} \mathrm{C} \pm 0.2^{\circ} \mathrm{C}$ to $33.1^{\circ} \mathrm{C} \pm 0.5^{\circ} \mathrm{C}$ (mean \pm standard deviation) in this procedure. No neurologic events were observed in any animal after the surgical procedures.

The global ischemia-reperfusion brains were evaluated for 3 purposes. First, 3 rats were killed 1 hour after reperfusion, and brain sections were observed with fluorescence microscopy to investigate the transfection of fluorescein isothiocyanate (FITC)-labeled ODN delivery. Second, 5 rats from each group were killed 1 hour after reperfusion, and the hippocampus, including the CA1 region, was collected to test the effect of transfected NF- $\kappa \mathrm{B}$ decoy ODNs on the expression of messenger RNAs that are known to be activated by NF- $\kappa$ B. The samples weighed 20 to $25 \mathrm{mg}$ after the blood vessels were stripped away. Third, 10 rats from each group were killed 7 days after the global brain ischemia for histologic study by means of terminal deoxynucleotidyl transferase-mediated dUTP nick end labeling (TUNEL) stain or immunohistochemistry for microtubule-associated protein 2 (MAP2) to investigate the neuronal damage.

\section{Quantification of TNF- $\alpha$, IL-1 $\beta$, and ICAM-1 mRNA Levels in the Hippocampus}

To clarify the effect of the in vivo transfection of the NF- $\kappa \mathrm{B}$ decoy ODNs versus the $\mathrm{S}$ decoy control ODNs on the expression of genes that are known to respond to the signal of NF- $\mathrm{KB} 1$ hour after reperfusion, we used a real-time polymerase chain reaction (PCR) system. This technique enables the quantification of complemen- 

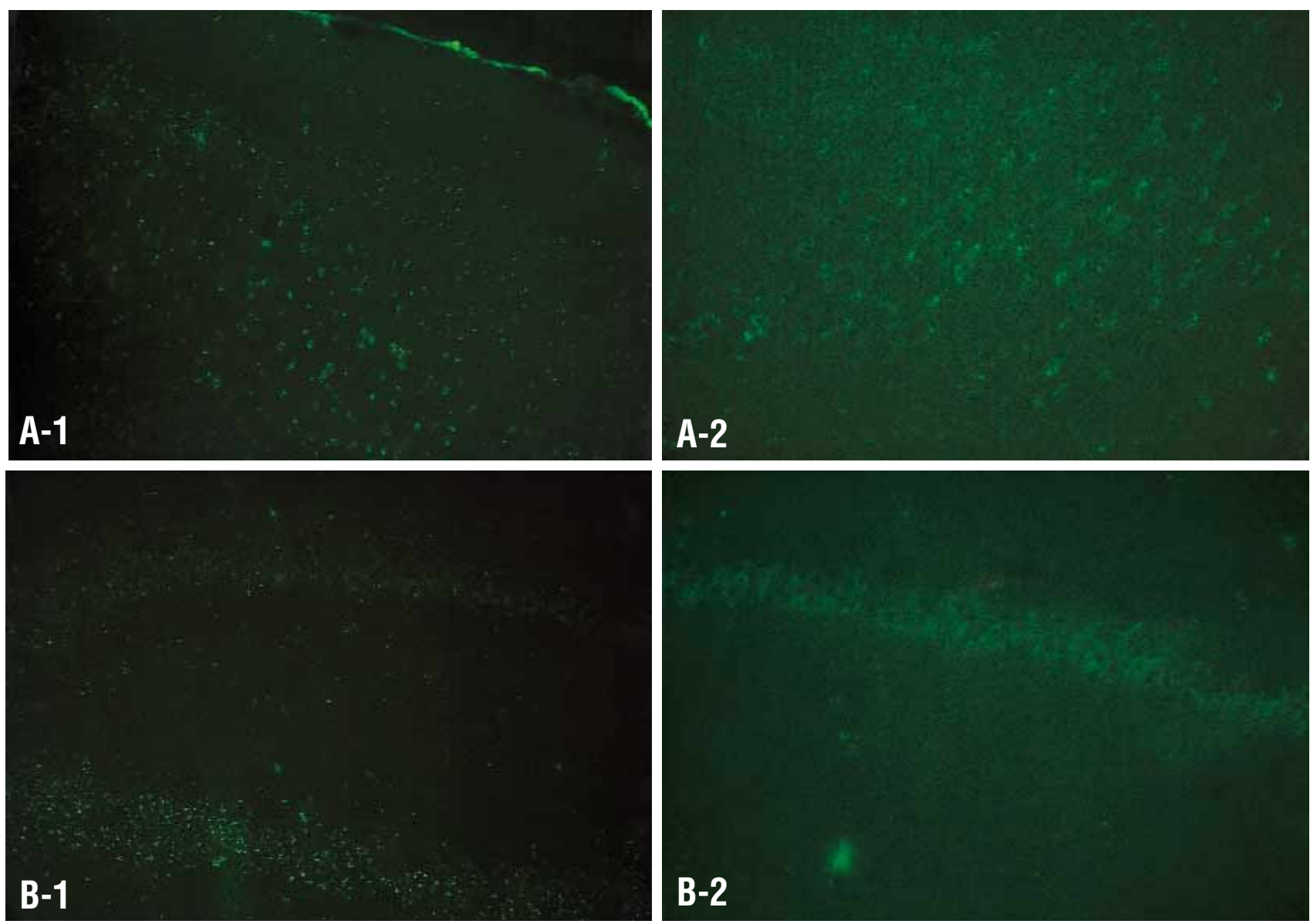

Figure 1. Transfection of rat tissue with FITC-labeled NF- $K B$ decoy ODNs 1 hour after reperfusion. FITC fluorescence was observed entirely and could be observed in nuclei in neurons throughout the brain. $A$, Rat cortex section; $\mathrm{B}$, hippocampus. (Original magnification $40 \times[A-1, B-1]$ or $100 \times[A-2, B-2]$.)

tary DNA amplification and involves fluorescence-based real-time PCR followed by measurement of the amplification with the ABI PRISM 7700 Sequence Detection System (Applied Biosystems, Foster City, Calif).

In brief, total RNA was purified from each 20- to 25-mg hippocampus sample by using the RNAeasy Mini Kit, according to the manufacturer's instructions (Qiagen, Hilden, Germany). The RNA samples were frozen in liquid nitrogen and stored at $-80^{\circ} \mathrm{C}$ until use. To test for gene transcription, $2 \mu \mathrm{g}$ of RNA was reverse transcribed with RNase-H-negative Moloney Mouse Leukemia Virus reverse transcriptase (SUPERSCRIPT 2, Gibco BRL, Life Technologies, Inc, Rockville, Md) in a total volume of $40 \mu \mathrm{L}$, as recommended by the manufacturer. One eightieth of the cDNA was used for each PCR reaction, and measurement of each transcript was performed in triplicate. The technique of real-time PCR is based on the hydrolysis of a specific fluorescent probe at each amplification cycle by the $5^{\prime}$-endonuclease activity of Taq polymerase. This technique was performed as described by Depre and colleagues, ${ }^{14}$ with some modification. The nucleotide sequences of the forward primers, reverse primers, and probes were as follows: TNF- $\alpha$, forward primer CCACCACGCTCTTCTGTCTACT, reverse primer TTGGTGGTTTGCGACGACGT, and probe CCCAGACCCTCACACTCAGATCATCTTC; IL-1 $\beta$, forward primer CCACCTCAATGGACAGAACATAAG, reverse primer GACAAACCGCTTTTCCATCTTC, and probe CAAGGAGAGACAAGCAACGACAAAATCCC; and ICAM-1, forward primer TTCAAGCTGAGCGACATTGG, reverse primer TCAGTGTCTCATTCCCACGGA, and probe, TCTGCCACCATCACTGTGTATTCGTTCC.

For each molecule assayed here, the primer pair, or at least one primer or probe, was designed to span one or more introns so that only mRNA would be measured. In fact, when genomic DNA was used as a target, no signal was detected for any of the molecules. Primers and probes were used at $200 \mathrm{nmol} / \mathrm{L}$ in each $\mathrm{PCR}$ reaction, and PCR was performed with 50 cycles of a 15 -second denaturing step at $95^{\circ} \mathrm{C}$ and a 1 -minute annealing step at $60^{\circ} \mathrm{C}$. The correlation coefficient of the standard curves generated in each measurement was always around 0.97 or better, and the coefficient of variance in the triplicate samples was usually less than $10 \%$. Because of the relative lack of precision in the measurement of RNA concentration with spectrophotometry, the level of transcripts for the cellular enzyme glyceraldehyde 3-phosphate dehydrogenase 

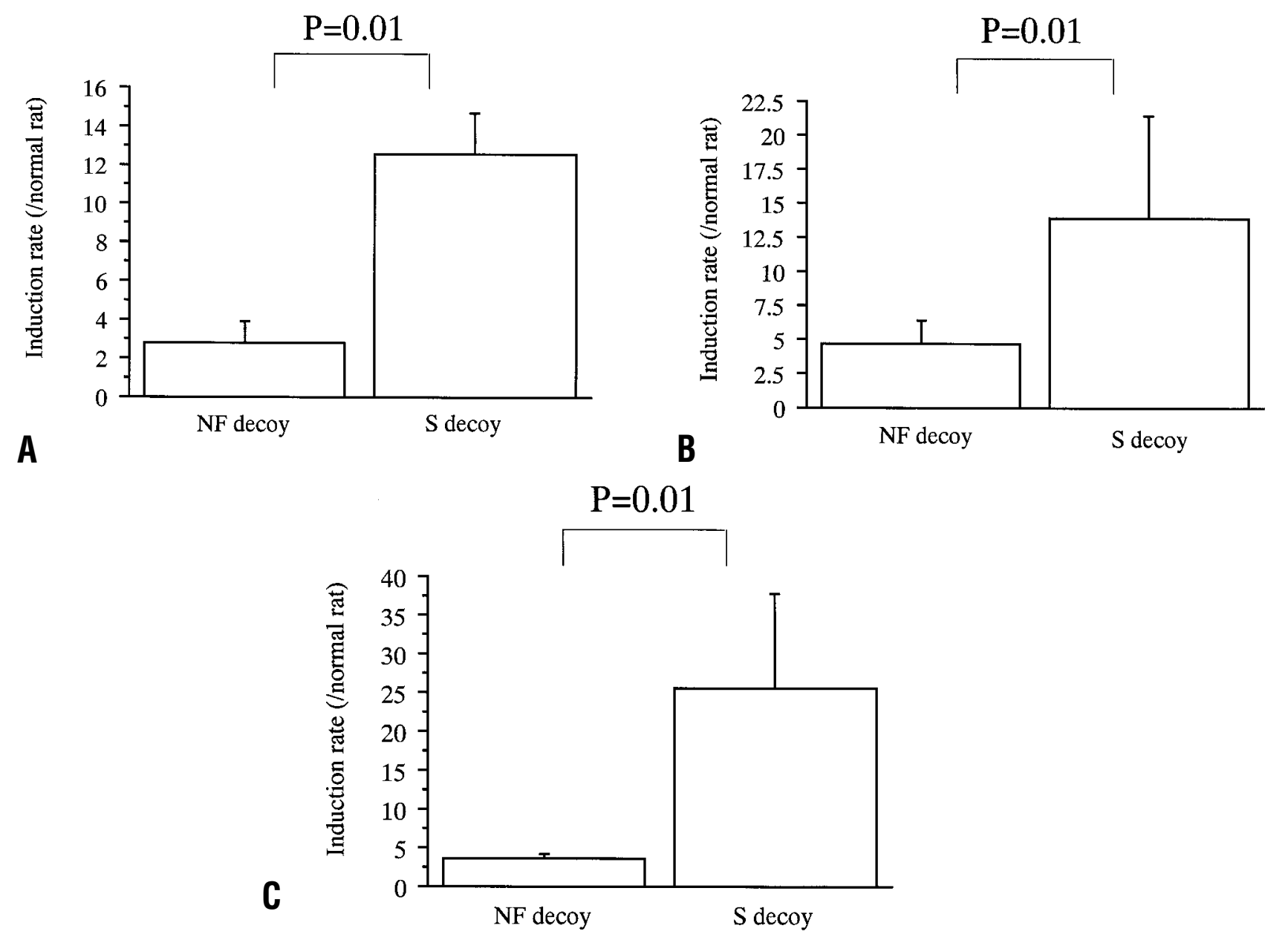

Figure 2. Induction rate of mRNA in the rat hippocampus 1 hour after reperfusion. The levels of these mRNAs were normalized to the mRNA levels of GAPDH in each sample, and the induction rates were calculated by comparing them with levels in normal rat hippocampus. A, TNF- $\alpha$ mRNA; B, IL-1 $\beta$ mRNA; C, ICAM-1 mRNA. All values were significantly suppressed in the NF- $\kappa B$ decoy group compared with in the $S$ decoy group $(P=.01)$.

(GAPDH) was quantitatively measured in each sample as the internal control. The GAPDH primer and probe sequences were as follows: forward primer, CCATCACTGCCACTCAGAAGAC; reverse primer, TCATACTTGGCAGGTTTCTCCA; and probe, CGTGTTCCTACCCCCAATGTATCCGT.

The mRNA/GAPDH value was calculated for each sample, and then the induction value compared with the normal rat mRNA/GAPDH level was calculated.

\section{TUNEL Staining and Immunohistochemistry}

In normal animals 1 week after global brain ischemia, ischemic damage can be detected, especially in the CA1 region of the hippocampus, and the number of TUNEL-positive neurons increases. ${ }^{1}$ The presence of TUNEL-positive neurons does not directly reveal the occurrence of apoptosis but does indicate DNA damage. The expression levels of MAP2, a cytoskeletal protein, is a neuronal marker of ischemic injury, and decreases in its expression have been observed after global brain ischemia. ${ }^{15}$ To assess the effect of transfecting the NF- $\mathrm{KB}$ decoy ODNs on neuronal ischemic injury, brains were rapidly frozen in liquid nitrogen and sectioned coronally through the rostrocaudal extent of the hippocampus. For TUNEL staining, $5-\mu \mathrm{m}$ sections were fixed in $1 \%$ paraformaldehyde. TUNEL staining was performed with the ApopTag In Situ Apoptosis Detection Kit (Intergen Co, Purchase, NY), as recommended by the manufacturer. The reaction product was visualized by development with 3,3'-diaminobenzidine and $\mathrm{H}_{2} \mathrm{O}_{2}$. TUNELstained brain sections were also stained with hematoxylin and eosin. Then the percentage of the total number of neurons that were TUNEL positive was calculated in the CA1 region $(500 \mu \mathrm{m}$ in length) in 3 sections in each rat.

Immunohistochemistry was performed on the brain sections by using the avidin-biotin peroxidase system (ABC kit; Vector Laboratories, Inc, Burlingame, Calif). Five-micrometer sections were fixed in $2 \%$ paraformaldehyde and incubated with a monoclonal MAP2 antibody (Upstate Biotechnology, Lake Placid, NY) overnight at $4^{\circ} \mathrm{C}$. Sections were stained by using the $\mathrm{ABC}$ immunoperoxidase system, according to the manufacturer's recommendations. The reaction product was visualized by develop- 

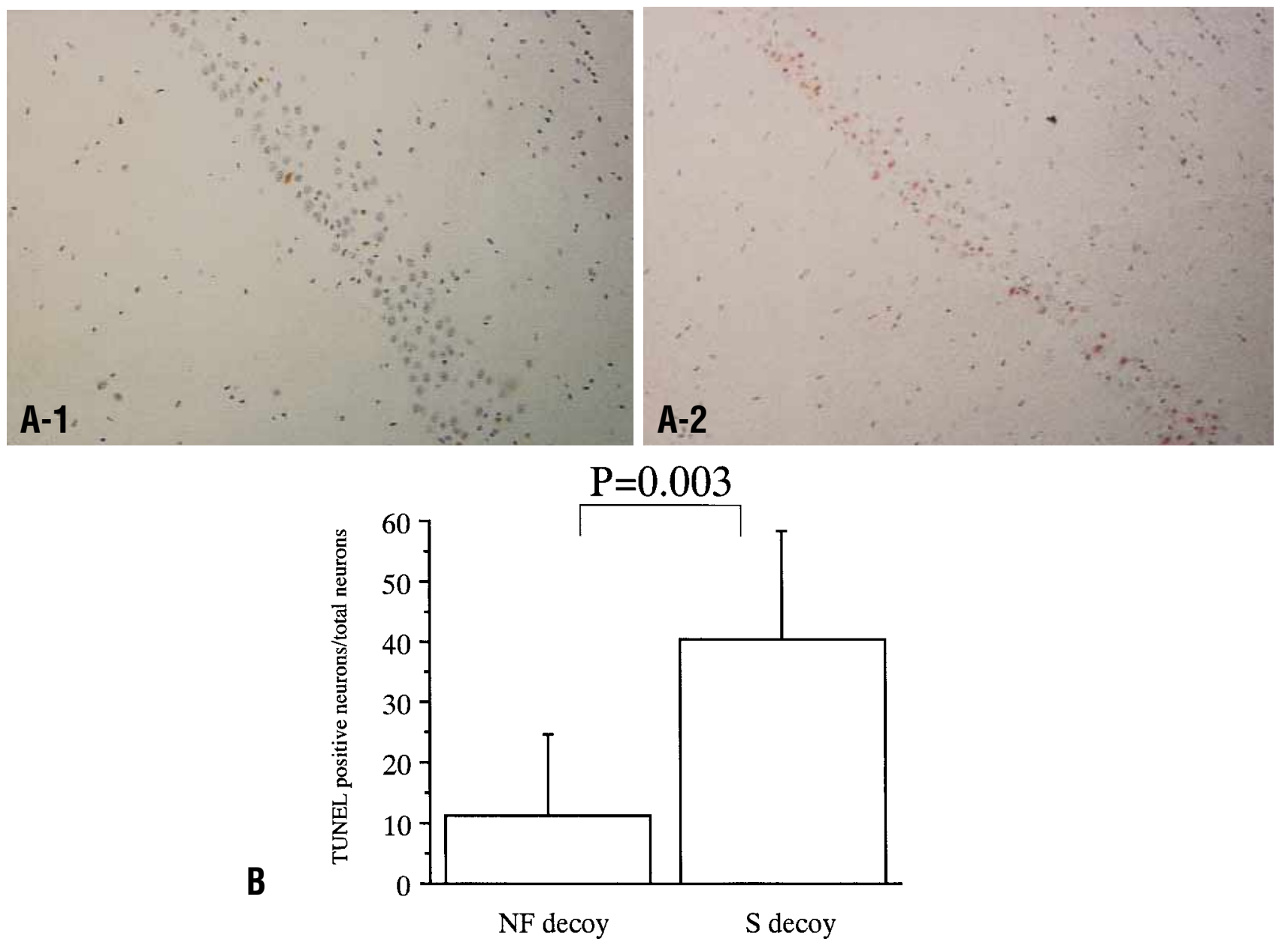

Figure 3. A, Sections through the rat hippocampus CA1 region with TUNEL stain 7 days after global brain ischemia. NF- $\kappa B$ decoy ODN therapy (A-1) suppressed the appearance of TUNEL-positive neurons (stained in brown) compared with in the $S$ decoy group $(A-2)$. B, Percentage of TUNEL-positive neurons in the hippocampus CA1 region (500 $\mu \mathrm{m}$ in length). The percentage of TUNEL-positive neurons significantly decreased in the NF- $\mathrm{B}$ decoy group compared with in the $S$ decoy group $(P<.01)$. (Original magnification $100 \times[A-1, A-2]$.)

ment with 3,3'-diaminobenzidine and $\mathrm{H}_{2} \mathrm{O}_{2}$, and these sections were also stained with hematoxylin and eosin. Then the number of MAP2-positive neurons was counted in the medial CA1 region (500 $\mu \mathrm{m}$ in length) in 3 sections in each rat.

\section{Statistical Analysis}

Data are presented as means \pm standard deviation. Statistically significant differences between the 2 groups were calculated with the Mann-Whitney $U$ test.

\section{Results}

\section{In Vivo Transfection of NF- $\kappa B$ Decoy ODNs Through} the Carotid Artery During Global Brain Ischemia

In our preliminary study we infused the naked FITC-labeled NF-KB ODNs into the carotid artery without any vector during global brain ischemia. However, fluorescence was not detected in the brain tissue by using this method (data not shown). We next tried using the HVJ-liposome method to transfect the NF- $\kappa \mathrm{B}$ decoy ODNs into brain tissue. One hour after reperfusion, in all of the rats examined, we observed the transfection of cells with the FITC-labeled ODNs not only in the intima of arteries but also in neurons, especially those of the cortex and hippocampus (Figure 1). The fluorescence was localized mainly to the cell nuclei. Thus the NF- $\mathrm{KB}$ decoy ODNs could be transfected into the brain tissue through the blood-brain barrier during global brain ischemia in our model.

TNF- $\alpha$, IL-1 $\beta$, and ICAM-1 mRNA Expression in the Hippocampus

In the NF decoy group the fold-induction rate of expression of the gene encoding TNF- $\alpha 1$ hour after reperfusion compared with that seen in normal rats was $2.8 \pm 1.1$, whereas the fold 

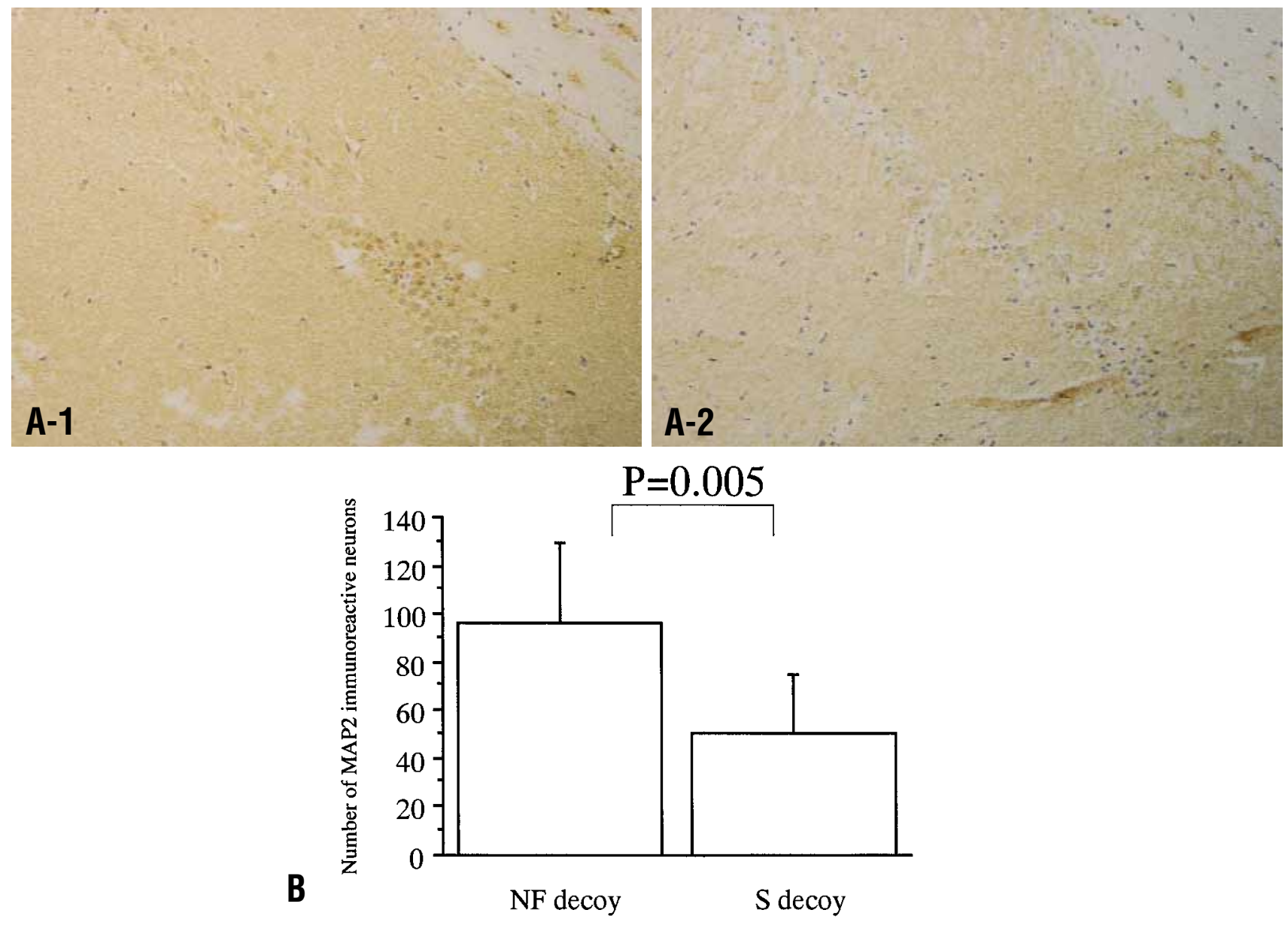

Figure 4. A, Sections of the rat hippocampus CA1 region with MAP2 immunohistologic stain 7 days after global brain ischemia. NF- $K B$ decoy ODNs therapy (A-1) suppressed the appearance of MAP2-negative neurons (without immunoreactivity in the cytosol) compared with in the $S$ decoy group (A-2). B, The number of MAP2-positive neurons in the hippocampus CA1 region (500 $\mu \mathrm{m}$ in length). The number of MAP2-positive neurons was significantly maintained in the NF decoy group compared with in the $S$ decoy group $(P<.01)$. (Original magnification $100 \times[A-1, A-2]$.)

induction was $12.5 \pm 2.2$ in the $\mathrm{S}$ decoy group. The fold-induction rates of IL-1 $\beta$ and ICAM-1 mRNA expression 1 hour after reperfusion were $4.7 \pm 1.7$ and $3.5 \pm 0.5$ in the NF decoy group and $14.0 \pm 7.5$ and $25.7 \pm 12.0$ in the $\mathrm{S}$ decoy group, respectively (Figure 2). The expression of these 3 genes is activated by NF- $\kappa \mathrm{B}$ and was effectively suppressed by the transfection of NF- $\mathrm{KB}$ decoy ODNs through the carotid artery $(P=$ $.01, P=.01$, and $P=.01$, respectively). These data suggested that the transfected NF-KB decoy ODNs effectively blocked gene expression related to the NF- $\mathrm{\kappa B}$ signal in ischemia-reperfusion injury in the hippocampus.

\section{Blockade of Neuronal Damage by NF- $\kappa$ B Decoy ODNs in the Hippocampus CA1 Region}

Brain tissue was evaluated histologically 7 days after global brain ischemia to determine the protective effect of NF$\kappa \mathrm{B}$ decoy ODNs against neuronal damage. TUNEL-positive neurons were detected in both hemispheres, and the neuronal damage was estimated in the right hemisphere.

In the NF decoy group, 7 days after global brain ischemia, there were fewer TUNEL-positive neurons, compared with in the S decoy group $(11.3 \% \pm 13.1 \%$ in the NF decoy group and $40.3 \% \pm 18.0 \%$ in the $\mathrm{S}$ decoy group, $P=$ .003; Figure 3). The number of MAP2-positive neurons was higher in the NF decoy group than in the $\mathrm{S}$ decoy group $(96.4 \pm 33.0$ cells $/ 500 \mu \mathrm{m}$ length in the NF decoy group and $50.6 \pm 23.8$ cells $/ 500 \mu \mathrm{m}$ length in the $\mathrm{S}$ decoy group, $P=$ .005 ; Figure 4). These data show that transfecting NF- $\mathrm{\kappa B}$ decoy ODNs into neurons through the carotid artery attenuated the neuronal damage after global brain ischemia.

\section{Discussion}

This study demonstrated evidence of attenuating neuronal damage after global brain ischemia by using in vivo trans- 
fection of a cis element decoy to bind the transcriptional factor NF- $\kappa \mathrm{B}$. NF- $\kappa \mathrm{B}$ decoy ODNs were successfully introduced into the nuclei of neurons by infusing them through the carotid artery and across the blood-brain barrier. The transfected NF- $\mathrm{KB}$ decoy ODNs suppressed gene expression related to NF- $\kappa \mathrm{B}$ signal in the hippocampus and attenuated neuronal damage caused by global brain ischemia, as assessed by TUNEL labeling (DNA fragmentation) and the immunoreactivity of MAP2 (neuronal marker). Thus, the transfection of neurons with NF- $\mathrm{KB}$ decoy ODNs through the carotid artery may provide a novel strategy to protect the brain against ischemic injury during global brain ischemia.

Deep hypothermia is a basic strategy for brain protection during circulatory arrest that reduces the cerebral energy requirements. However, deep hypothermic circulatory arrest carries an adverse risk of neurologic injury and is associated with complications, such as seizures, cerebral palsy, motor dysfunction, and memory deficits. ${ }^{16-18}$ Neuronal damage, including necrosis and delayed neuronal death, is one cause of these neurologic injuries. In this study no neurologic event was revealed in rats, and histologic study showed no infarction area in the brain section. However, we speculate that the rats 7 days after ischemia in the control group may have had possibly impaired learning ability compared with those in the NF- $\mathrm{BB}$ decoy group, although all animals survived. Several methods in addition to deep hypothermia have been reported to attenuate the neuronal damage. ${ }^{19}$ However, these reports have mainly focused on the regulation of energy requirements and metabolism, and they have generally failed to prove the clinical success. Therefore, alternative methods on the basis of other mechanisms, such as the regulation of gene expression related to ischemia-reperfusion injury, seem warranted.

Recent reports have demonstrated that apoptosis may play an important role in delayed neuronal damage after circulatory arrest. ${ }^{20,21}$ Many molecular signals, including the inflammation-related cytokines and adhesion molecules, are involved in apoptosis. These inflammation-related agents are upregulated mainly by the transcriptional activation of $\mathrm{NF}-\kappa \mathrm{B}$, which is an oxidative-stress responsive signal. It is unclear whether the regulation of cytokine mRNA levels, such as TNF- $\alpha$ and IL- $1 \beta$, directly blocks the neuronal damage. However, at a minimum, these inflammatory cytokines seem to contribute the ischemia-related neuronal damage. Our data suggest that NF- $\kappa \mathrm{B}$ may play an important role in the attenuation of ischemia-reperfusion injury and neuronal damage after global brain ischemia. TUNEL staining is a nonspecific technique that may show DNA injury and repair of DNA and may even be positive in necrotic cells. Therefore, we are cautious to overstate apoptosis in TUNEL-positive neurons. However, histologic examination showed that TUNEL-positive neurons were about $15 \%$ in total neurons (fewer than TUNEL-positive neurons 7 days after global brain ischemia) 2 days after global brain ischemia (data were not shown), and the neuronal damage, including both necrosis and delayed neuronal death, should occur at least between 2 days and 7 days after global brain ischemia.

In addition, NF- $\mathrm{\kappa B}$ has been speculated to work through a number of pathways, and these might also induce neuronal damage after global brain ischemia. These mechanisms are as follows: (1) NF- $\mathrm{\kappa B}$ activation may partially mediate freeradical damage in several tissues, including the brain ${ }^{22}$; (2) NF- $\mathrm{KB}$ activation seems to cause glutamate cytotoxicity ${ }^{9}$; (3) NF- $\kappa B$ is likely to be instrumental in the upregulation of inducible nitric oxide synthase and cyclooxygenase $2^{23}$; and (4) NF- $\mathrm{KB}$ may mediate the activation of the CD95 ligand, which initiates delayed neuronal damage. ${ }^{24}$ These mechanisms are all potential targets of the NF- $\kappa \mathrm{B}$ decoy ODN method. Moreover, target genes for NF- $\mathrm{KB}$ include the apoptosis-related genes, including $T P 53^{25}$ and c-myc. ${ }^{26}$ Therefore, gene therapy with decoy ODNs against the NF$\kappa \mathrm{B}$ binding site seemed a likely method to provide a new strategy for neuronal protection during ischemia by suppressing the gene expression related to the inflammatory response and the subsequent neuronal damage, including apoptosis.

Many investigators have reported strategies using gene therapy for brain protection. ${ }^{27,28}$ In these reports genes were injected into the subarachnoid space or brain ventricles. However, no study has shown effective gene transfection by means of injection through the carotid arteries because the blood-brain barrier prevents the entry of many foreign substances and microorganisms. During and after global brain ischemia, however, permeability across the blood-brain barrier increases; in fact, relief of the blood-brain barrier has been reported to extend for up to 6 hours after ischemia. ${ }^{29}$ We believe that our success in transfecting neurons with the decoy ODNs was due to the accumulation of the HVJ-liposome complex in the brain tissue. To the best of our knowledge, this is the first report of successful gene transfection through the carotid arteries and across the blood-brain barrier. Further study is needed to clarify the mechanism of gene transfer under these conditions.

Decoy therapy has many benefits, including immediate effect, low cost, and few complications, and gene therapy with naked E2F decoy has already been attempted in the clinical setting to prevent vein graft disease. ${ }^{30}$ However, transfection of naked ODNs has limitations in its efficiency in the brain through the carotid artery in our preliminary study. On the other hand, more safety vectors are in the process of development. Therefore, clinical application of NF- $\kappa$ B decoy therapy with HVJ-liposome or other vectors through the carotid artery may be possibly attempted for brain protection against ischemic injury during circulatory arrest in the near future. NF- $\kappa \mathrm{B}$ decoy ODN therapy 
through vessels might have a potential of wide application in clinical use for brain protection, such as retrograde perfusion of cerebroplegia.

In summary, the results of this study indicate that administration of $\mathrm{NF}-\kappa \mathrm{B}$ decoy ODNs during global brain ischemia attenuates neuronal damage in the hippocampus $\mathrm{CA} 1$ region in a rat model. Thus NF- $\mathrm{KB}$ decoy ODN administration through the carotid artery can protect neurons during global brain circulatory arrest, raising the possibility that NF- $\mathrm{KB}$ decoy ODNs may become a promising therapeutic agent for protecting the brain against global ischemia. We believe that this method of gene transfection to the brain might be applied not only in the field of cardiac surgery but also in other fields, such as neurosurgery.

We thank Akiko Nishimura for production of the HVJ-liposome complex and Shigeru Matsumi for animal care.

\section{References}

1. Jonas RA. Hypothermia, circulatory arrest, and the pediatric brain. $J$ Cardiothorac Vasc Anesth. 1996;10:66-74.

2. Kirino T. Delayed neuronal death in the gerbil hippocampus following ischemia. Brain Res. 1982;239:57-69.

3. Kirklin JW, Barratt-Boyes BG. Hypothermia, circulatory arrest, and cardiopulmonary bypass. In: Kirklin JW, Barratt-Boyes BG, editors. Cardiac surgery. New York: Churchill Livingstone; 1993. p. 66-73.

4. Stephenson D, Yin T, Smalstig EB, Hsu MA, Panetta J, Little S, et al. Transcription factor nuclear factor-kappa $\mathrm{B}$ is activated in neurons after focal cerebral ischemia. J Cereb Blood Flow Metab. 2000;20:592-603.

5. Schneider A, Martin-Villalba A, Weih F, Vogel J, Wirth T, Schwaninger M. NF-kappa B is activated and promotes cell death in focal cerebral ischemia. Nat Med. 1999;5:554-9.

6. Clemens JA, Stephenson DT, Dixon EP, Smalstig EB, Mincy RE, Rash $\mathrm{KS}$, et al. Global cerebral ischemia activates nuclear factor-kappa B prior to evidence of DNA fragmentation. Brain Res Mol Brain Res. 1997;48:187-96.

7. Christmann JW, Lancaster LH, Blackwell TS. Nuclear factor $\kappa$ B: a providal role in the systemic inflammatory response syndrome and new target for therapy. Intensive Care Med. 1998;24:1131-8.

8. Howard EF, Chen Q, Cheng C, Carroll JE, Hess-D. NF-kappa B is activated and ICAM-1 gene expression is upregulated during reoxygenation of human brain endothelial cells. Neurosci Lett. 1998;248:199-203

9. Grilli M, Pizzi M, Memo M, Spano P. Neuroprotection by aspirin and sodium salicylate through blockade of NF-KB activation. Science. 1996;274:1383-5.

10. Tomita N, Morishita R, Tomita S, Gibbons GH, Zhang L, Horiuchi M, et al. Transcription factor decoy for NF kappa B inhibits TNF-alphainduced cytokine and adhesion molecule expression in vivo. Gene Ther. 2000; 7:1326-32.

11. Morishita R, Sugimoto T, Aoki M, Kida I, Tomita N, Moriguchi A, et al. In vivo transfection of cis element "decoy" against nuclear factorkappa B binding site prevents myocardial infarction. Nat Med. 1997;3:894-9.

12. Sawa Y, Morishita R, Suzuki K, Kagisaki K, Kaneda Y, Maeda-K, et al. A novel strategy for myocardial protection using in vivo transfection of cis element "decoy" against NF kappa B binding site: evidence for a role of NF kappa B in ischemia-reperfusion injury. Circulation. 1997;96(Suppl 9): II-280-5.

13. Torre JC, Fortin T. Partial or global rat brain ischemia: the SCOT model. Brain Res Bull. 1991;26:365-72.

14. Depre C, Shipley GL, Chen W, Han Q, Doenst T, Moore M, et al. Unloaded heart in vivo replicates fetal gene expression of cardiac hypertrophy. Nat Med. 1998;4:1269-75.

15. Vanickey I, Balchen T, Diemer NH. Alterations in MAP2 immunostainability after prolonged complete brain ischemia in the rat. Neuroreport. 1995;7:161-4.

16. Rappaport LA, Wypij D, Bellinger DC, Helmers SL, Holmes GL, Barnes PD, et al. Relation of seizures after cardiac surgery in early infancy to neurodevelopmental outcome. Boston Circulatory Arrest Study Group. Circulation. 1998;97:773-9.

17. Bellinger DC, Jonas RA, Rappaport LA, Wypij D, Wernovsky G, Kuban KC, et al. Developmental and neurologic status of children after heart surgery with hypothermic circulatory arrest or low-flow cardiopulmonary bypass. N Engl J Med. 1995;332:549-55.

18. Reich DL, Uysal S, Sliwinski M, Ergin MA, Kahn RA, Konstadt SN, et al. Neuropsychologic outcome after deep hypothermic circulatory arrest in adults. J Thorac Cardiovasc Surg. 1999;117:156-63.

19. Aoki M, Jonas RA, Nomura F, Stromski ME, Tsuji MK, Hickey PR, et al. Effects of cerebroplegic solutions during hypothermic circulatory arrest and short-term recovery. $J$ Thorac Cardiovasc Surg. 1994;108:291-301.

20. Cheng Y, Deshumukh M, D'Costa M, Demaro JA, Gidday JM, Shah A, et al. Caspase inhibitor affords neuroprotection with delayed administration in a rat model of neonatal hypoxic-ischemic injury. $J$ Clin Invest. 1998;101:1992-9.

21. Kurth CD, Priestley M, Golden J, McCann J, Raghupathi R. Regional patterns of neuronal death after deep hypothermic circulatory arrest in newborn pigs. J Thorac Cardiovasc Surg. 1999;118:1068-77.

22. Schreck R, Rieber P, Baeuerle PA. Reactive oxygen intermediates as apparently widely used messengers in the activation of the NF- $\mathrm{KB}$ transcription factor and HIV-1. EMBO J. 1991;10:2247-58.

23. Schulze-Osthoff K, Ferrari D, Riehemann K, Wesselborg S. Regulation of NF- $\mathrm{KB}$ activation by MAP kinase cascades. Immunobiology. 1997;198:35-49.

24. Vogt M, Bauer MK, Ferarri D, Schulze-Osthoff K. Oxidative stress and hypoxia/reoxygenation trigger CD95 (Apo-1/Fas) ligand expression in microglial cells. FEBS Lett. 1998;429:67-72.

25. Wu H, Lozano G. NF- $\kappa B$ activation of $\mathrm{p} 53$ : a potential mechanism for suppressing cell growth in response to stress. J Biol Chem. 1994;269:20067-74.

26. LaRosa FA, Pierce JW, Sonenshein GE. Differential regulation of the c-myc oncogene promoter by the NF- $\mathrm{KB}$ Rel family of transcription factors. Mol Cell Biol. 1994;14:1039-44.

27. Hagihara Y, Saitoh Y, Kaneda Y, Kohmura E, Yoshimine T. Widespread gene transfection into the central nervous system of primates. Gene Ther. 2000;7:759-63.

28. Ono S, Date I, Onoda K, Shiota T, Ohmoto T, Ninomiya Y, et al. Decoy administration of NF-kappa B into the subarachnoid space for cerebral angiopathy [published erratum appears in Hum Gene Ther 1999;10:335].

29. Preston E, Foster DO. Evidence for pore-like opening of the bloodbrain barrier following forebrain ischemia in rats. Brain Res. 1997;761:4-10.

30. Mann MJ, Whittemore AD, Donaldson MC, Belkin M, Conte MS, Polak JF, et al. Ex-vivo gene therapy of human vascular bypass grafts with E2F decoy: the PREVENT single-centre, randomised, controlled trial. Lancet. 1999;354:1493-8. 